\title{
A NOTE ON A SYSTEM OF EQUATIONS WITH INFINITELY MANY UNKNOWNS*
}

\author{
BY L. W. COHEN $\dagger$
}

If the system of equations

$$
x_{i}+\sum_{k=1}^{\infty} a_{i k} x_{k}=y_{i}
$$

is considered as a transformation on the space $\left(x_{k}\right)$ such that $\sum_{k=1}^{\infty}\left|x_{k}\right|^{p}$ converges for some $p>1$, then we must require that $\sum_{k=1}^{\infty}\left|a_{i k}\right|^{p /(p-1)}$ converges for every $i$. If we further require that this transformation carry $\left(x_{k}\right)$ into $\left(y_{k}\right)$ of the same space, we are led to consider the inequality

$$
\begin{aligned}
{\left[\sum_{i=1}^{\infty}\left|y_{i}\right|^{p}\right]^{1 / p} \leqq } & {\left[\sum_{i=1}^{\infty}\left|x_{i}\right|^{p}\right]^{1 / p} } \\
& \cdot\left[1+\left\{\sum_{i=1}^{\infty}\left[\sum_{k=1}^{\infty}\left|a_{i k}\right|^{p /(p-1)}\right]^{p-1}\right\}^{1 / p}\right]
\end{aligned}
$$

obtained from (1) by the Hölder and Minkowski inequalities. $\S$ The convergence of the double series on the right is a sufficient condition. Since $p$ and $p /(p-1)$ are separated by 2 if not equal to it, we will consider $p \leqq 2$. It will appear in IV below that in the case $p \geqq 2$, the matrix of the transformation is the transposed of that of (1). For the system (1), under the condition that

* Presented to the Society, October 26, 1929.

† National Research Fellow.

¥Landau, Über einen Konvergentzsatz, Göttinger Nachrichten, 1907, pp. 25-27.

$\S$ These are respectively

and

$$
\sum\left|a_{n} b_{n}\right| \leqq\left[\sum\left|a_{n}\right|^{p}\right]^{1 / p}\left[\sum\left|b_{n}\right|^{p /(p-1)}\right]^{(p-1) / p}
$$

$$
\left[\sum\left|a_{n}+b_{n}\right|^{p}\right]^{1 / p} \leqq\left[\sum\left|a_{n}\right|^{p}\right]^{1 / p}+\left[\sum\left|b_{n}\right|^{p}\right]^{1 / p}, \quad(p>1) .
$$

See F. Riesz, Les Systèmes d'Équations Linéaires . . , Paris, 1913, p. 45. 


$$
\sum_{i=1}^{\infty}\left[\sum_{k=1}^{\infty}\left|a_{i k}\right|^{p /(p-1)}\right]^{p-1} \text { converges, } \quad(1<p \leqq 2),
$$

we state the following theorems.

TheOREM 1. The system (1) is equivalent to (that is, has the same solution as) one which has an absolutely convergent determinant $\Delta$ in the sense of von Koch.

THEOREM 2. If $\Delta \neq 0$ and $\sum_{i=1}^{\infty}\left|y_{i}\right|^{p}$ converges, then the solution is obtainable from the new system by Cramer's rule and is in the same space.

THEOREM 3. The inverse of (1) is equivalent to a system with an absolutely convergent determinant whose elements satisfy (3).

THEOREM 4. If (1) is transposed, it becomes equivalent to a system with an absolutely convergent determinant and transforms the space $\left(x_{k}\right)$, where $\sum_{k}\left|x_{k}\right|^{p /(p-1)}$ converges, $(p /(p-1) \geqq 2)$, into the same space. The transposed system has the properties of Theorems 2 and 3 with $p$ replaced by $p /(p-1)$ and $i$ and $k$ interchanged.

Theorems 1 and 2 are slight extensions of results obtained by Bóbr, ${ }^{*}$ while 3 and 4 seem to be new.

We first establish the following lemma.

LEMmA. If, in the determinant $\Delta=\left|\delta_{i k}+a_{i k}\right|,(i, k=1,2, \cdots)$, $\sum_{i=1}^{\infty}\left|a_{i i}\right|$ and $\sum_{i=1}^{\infty}\left[\sum_{k=1}^{\infty}\left|a_{i k}\right|^{p /(p-1)}\right]^{p-1},(i \neq k ; 1<p \leqq 2)$, converge, then

$$
\begin{aligned}
& \sum_{k=1}^{\infty}\left[\sum_{i=1}^{\infty}\left|a_{i k}\right|^{p}\right]^{1 /(p-1)}, \\
& \sum_{k=1}^{\infty}\left[\sum_{i=1}^{\infty}\left|A_{i k}\right|^{p /(p-1)}\right]^{p-1}, \quad(i \neq k), \\
& \sum_{i=1}^{\infty}\left[\sum_{k=1}^{\infty}\left|A_{i k}\right|^{p}\right]^{1 /(p-1)}
\end{aligned}
$$

converge and $\lim _{i} A_{i i}=\Delta$, where $A_{i k}$ is the cofactor of $a_{i k}$.

* Bóbr, Eine Verallgemeinerung des v. Kochschen Satzes ..., Mathematische Zeitschrift, vol. 10 (1921), pp. 1-11. Bobr's conditions on (1) are that $\sum_{i=1}^{\infty}\left|a_{i i}\right|$ and $\sum_{i=1}^{\infty}\left[\sum_{k=1}^{\infty}\left|a_{i k}\right| p /(p-1)\right]^{p-1}(i \neq k ; 1<p \leqq 2)$ converge. The absolute convergence of the determinant is established and the solution is obtained by indirect methods involving the theory of limited linear and bilinear forms. 
Proof: The determinant and its minors converge absolutely and the following relations hold :*

$$
\begin{aligned}
& \sum_{i=1}^{\infty}\left[\sum_{k=1}^{\infty}\left|a_{i k}\right|^{p /(p-1)}\right]^{p-1}=\sigma^{p} \\
& \sum_{k=1}^{\infty}\left[\sum_{i=1}^{\infty}\left|a_{i k}\right|^{p}\right]^{1 /(p-1)}=\tau^{p /(p-1)} \leqq \sigma^{p /(p-1)}, \quad(i \neq k) \\
& \sum_{i_{n+1}=1}^{\infty}\left[\sum_{i_{2}, \cdots, i_{n}=1}^{\infty}\left|i_{1}, i_{2}, \cdots, i_{n} ; i_{n+1}\right|\right]^{p /(p-1)} \\
& \leqq \tau^{(n-1) p /(p-1)} \sum_{i_{n+1}=1}^{\infty}\left|i_{1} ; i_{n+1}\right| p /(p-1) \\
& \sum_{i_{1}=1}^{\infty}\left[\sum_{i_{2}, \ldots, i_{n}=1}^{\infty} \mid i_{1}, i_{2}, \cdots, i_{n} ; i_{n+1}\right]^{p}
\end{aligned}
$$$$
\leqq \sigma^{(n-1) p} \sum_{i_{1}=1}^{\infty}\left|i_{1}, i_{n+1}\right|^{p},
$$

where $\left|i_{1}, i_{2}, \cdots, i_{n} ; i_{n+1}\right|=\left|a_{i_{1} i_{2}} a_{i_{2} i_{3}} \cdots a_{i_{n} i_{n+1}}\right|$ if all the subscripts are distinct and is zero otherwise.

By the interchange of a finite number of rows or columns in $A_{i k}$, its diagonal can be made to differ from that of $\Delta$ in that $1+a_{i i}$, and $1+a_{k k}$ are missing and $a_{k i}$ occurs. Then $A_{i k}$ may be expanded as a sum of infinite products of the form

$$
c_{1: 1} c_{2 j_{2}} \cdots c_{m j_{m}} \prod_{\mu=m+1}^{\infty} c_{\mu \mu},
$$

where $c_{h j}$ is the general term of $A_{i k},\left(j_{1}, j_{2}, \cdots, j_{m}\right)$ is a permutation of $(1,2, \cdots, m)$ and the $c_{\mu \mu}$ are on the diagonal of $A_{i k}$. If we replace, in each product*, $c_{h j}$ by $\delta_{h j}+a_{h j}$ and expand, we obtain new products in the $a_{h j}$. Each of these except for sign is a product of $a_{k i}$ or $\left|k, i_{i}, \cdots, i_{n} ; i\right|(n \geqq 1)$ multiplied by a product of circular products of $\Delta . \dagger$ If we put $P=$ $\Pi(1+|\pi|)$, where the product is extended over all circular products of $\Delta$, we may write, $P$ being finite,

* Bobr, loc. cit.

† See F. Riesz, loc. cit., pp. 33-35. 
(12) $\left|A_{i k}\right| \leqq P\left\{\left|a_{k i}\right|+\sum_{n=1}^{\infty}\left(\sum_{i_{1}, \ldots, i_{n}=1}^{\infty}\left|k, i_{1}, \ldots, i_{n} ; i\right|\right)\right\}$, $(i \neq k)$. Using the Hölder inequality and (10), we obtain

$$
\begin{aligned}
& \sum_{i_{1}, \cdots, i_{n=1}}^{\infty}\left|k, i_{1}, i_{2}, \cdots, i_{n} ; i\right| \\
= & \sum_{i_{1}=1}^{\infty}\left|k ; i_{1}\right| \sum_{i_{2}, \cdots, i_{n=1}}^{\infty}\left|i_{1}, i_{2}, \cdots, i_{n} ; i\right| \\
\leqq & {\left[\sum_{i_{1}=1}^{\infty}\left|k ; i_{1}\right| p /(p-1)\right]^{(p-1) / p} } \\
\cdot & {\left[\sum_{i_{1}=1}^{\infty}\left\{\sum_{i_{2}, \cdots, i_{n=1}}^{\infty}\left|i_{1}, i_{2}, \cdots, i_{n} ; i\right|\right\}^{p}\right]^{1 / p} } \\
\leqq & \sigma^{n-1}\left[\sum_{i_{1}=1}^{\infty}\left|k ; i_{1}\right|^{p /(p-1)}\right]^{(p-1) / p}\left[\sum_{i_{1}=1}^{\infty}\left|i_{1} ; i\right|^{p}\right]^{1 / p} .
\end{aligned}
$$

Combining this with (12) and making the assumption that $\sigma<1$, we have

$$
\begin{aligned}
& \left|A_{i k}\right| \leqq P\left\{\left|a_{k i}\right|\right. \\
& \left.+\frac{1}{1-\sigma}\left[\sum_{i_{1}=1}^{\infty}\left|k ; i_{1}\right|^{p /(p-1)}\right]^{(p-1) / p}\left[\sum_{i_{1}=1}^{\infty}\left|i_{1} ; i\right|^{p}\right]^{1 / p}\right\} \\
& (k \neq i) .
\end{aligned}
$$

Let us consider a fixed $k$ and apply the Minkowski inequality:

$$
\begin{aligned}
& \text { (15) }\left[\sum_{i=1}^{\infty}\left|A_{i k}\right|^{p /(p-1)}\right]^{(p-1) / p} \leqq P\left(\sum _ { i = 1 } ^ { \infty } \left\{\left|a_{k i}\right|\right.\right. \\
& \left.\left.+\frac{1}{1-\sigma}\left[\sum_{i_{1}=1}^{\infty}\left|k ; i_{1}\right|^{p /(p-1)}\right]^{(p-1) / p}\left[\sum_{i_{1}=1}^{\infty}\left|i_{1} ; i\right|^{p}\right]^{1 / p}\right\}^{p /(p-1)}\right)^{(p-1) / p} \\
& \leqq P\left\{\sum_{i=1}^{\infty}\left|a_{k i}\right|^{p /(p-1)}\right\}^{(p-1) / p} \\
& +\frac{P}{1-\sigma}\left[\sum_{i_{1}=1}^{\infty}\left|k ; i_{1}\right|^{p /(p-1)}\right]^{(p-1) / p}\left\{\sum_{i=1}^{\infty}\left[\sum_{i_{1}=1}^{\infty}\left|i_{1} ; i\right|^{p}\right]^{1 /(p-1)}\right\}^{(p-1) / p} .
\end{aligned}
$$


Since $\left|k ; i_{1}\right|=\left|a_{k i_{1}}\right|$ or 0 according as $i_{1} \neq$ or $=k$, we have, using (8),

$$
\begin{aligned}
& {\left[\sum_{i=1}^{\infty}\left|A_{i k}\right|^{p /(p-1)}\right]^{(p-1) / p}} \\
& \quad \leqq\left(P \frac{1-\sigma+\tau}{1-\sigma}\right)\left\{\sum_{i=1}^{\infty}\left|a_{k i}\right|^{p /(p-1)}\right\}^{(p-1) / p}, \quad(i \neq k) .
\end{aligned}
$$

Then raising to the $p$ th power, summing as to $k$ and using ( 7 ), we have

$$
\left.\begin{array}{rl}
\sum_{k=1}^{\infty}\left[\sum_{i=1}^{\infty}\left|A_{i k}\right|^{p /(p-1)}\right]^{p-1} & \\
& \leqq \\
& \leqq\left(P \frac{1-\sigma+\tau}{1-\sigma}\right)^{p} \sum_{k=1}^{\infty}\left[\sum_{i=1}^{\infty}\left|a_{k i}\right|^{p /(p-1)}\right]^{p-1} \\
1-\sigma & 1-\sigma+\tau
\end{array}\right)^{p}, \quad(i \neq
$$

If we set $\left|k, i_{1}, \cdots, i_{n-1}, i_{n} ; i\right|=\left|i_{n} ; i\right|\left|k, i_{1}, \cdots, i_{n-1} ; i_{n}\right|$ and use (9), we are led to

$$
\sum_{i=1}^{\infty}\left[\sum_{k=1}^{\infty}\left|A_{i k}\right|^{p}\right]^{1 /(p-1)} \leqq\left(P \tau \frac{1-\tau+\sigma}{1-\tau}\right)^{p /(p-1)}(k \neq i)
$$

Hence we have established (5) and (6) in case $\sigma<1$. This restriction may be removed as follows. Since

$$
\sum_{i=1}^{\infty}\left[\sum_{k=1}^{\infty}\left|a_{i k}\right|^{p /(p-1)}\right]^{p-1}, \quad(k \neq i),
$$

converges, there is an integer $m$ such that

$$
\sum_{i=m+1}^{\infty}\left[\sum_{k=1}^{\infty}\left|a_{i k}\right|^{p /(p-1)}\right]^{p-1}<\frac{1}{2}, \quad(k \neq i) .
$$

There is a number $t(0<t<1)$ such that 


$$
t^{p} \sum_{i=1}^{m}\left[\sum_{k=1}^{\infty}\left|a_{i k}\right|^{p /(p-1)}\right]^{p-1}<\frac{1}{2}
$$

If we multiply the first $m$ rows of $\Delta$ by $t$, then in the new determinant $\Delta^{\prime}, \sigma<1$ and (5) and (6) hold for $\Delta^{\prime}$. Both determinants and their minors are absolutely convergent. If the $A_{i k}^{\prime}$ are the cofactors of the elements of $\Delta^{\prime}$, they differ from the $A_{i k}$ in that the first $m$ or $m-1$ rows are multiplied by $t$. But $t$ being less than 1 , we have in any case

$$
t^{m}\left|A_{i k}\right| \leqq\left|A_{i k}^{\prime}\right|
$$

and consequently

$$
\begin{aligned}
& \sum_{k=1}^{\infty}\left[\sum_{i=1}^{\infty}\left|A_{i k}\right|^{p /(p-1)}\right]^{p-1} \\
& \leqq t^{-m p} \sum_{k=1}^{\infty}\left[\sum_{i=1}^{\infty}\left|A_{i k}^{\prime}\right|^{p /(p-1)}\right]^{p-1}, \quad(i \neq k), \\
& \sum_{i=1}^{\infty}\left[\sum_{k=1}^{\infty}\left|A_{i k}\right|^{p}\right]^{1 /(p-1)}, \\
& \leqq t^{-m p /(p-1)} \sum_{i=1}^{\infty}\left[\sum_{k=1}^{\infty}\left|A_{i k}^{\prime}\right|^{p}\right]^{1 /(p-1)},
\end{aligned}
$$

so that (5) and (6) hold in general.

Finally we show that $\lim _{i} A_{i i}=\Delta$. Since $\Delta$ is absolutely convergent, we have $\Delta=\sum_{k=1}^{\infty}\left(\delta_{i k}+a_{i k}\right) A_{i k}$ for all $i$. From the Hölder inequality we get

$$
\begin{aligned}
& \left|\Delta-\left(1+a_{i i}\right) A_{i i}\right| \leqq \sum_{k=1}^{\infty}\left|a_{i k} A_{i k}\right| \\
& \leqq\left[\sum_{k=1}^{\infty}\left|a_{i k}\right|^{p /(p-1)}\right]^{(p-1) / p}\left[\sum_{k=1}^{\infty}\left|A_{i k}\right|^{p}\right]^{1 / p}, \quad(k \neq i) .
\end{aligned}
$$

Applying the inequality $A^{1 / p} B^{(p-1) / p} \leqq(1 / p) A+[(p-1) / p] B$, $(A \geqq 0, B \geqq 0, p>1)$, we have 


$$
\begin{aligned}
& \left|\Delta-\left(1+a_{i i}\right) A_{i i}\right| \leqq(1 / p)\left[\sum_{k=1}^{\infty}\left|a_{i k}\right|^{p /(p-1)}\right]^{p-1} \\
& +[(p-1) / p]\left[\sum_{k=1}^{\infty}\left|A_{i k}\right|^{p}\right]^{1 /(p-1)}, \quad(k \neq i) .
\end{aligned}
$$

Now in consequence of (7), (22), and the fact that $\lim _{i} a_{i i}=0$, we have

$$
\lim _{i} A_{i i}=\Delta
$$

We return now to our system (1) under condition (3).

I. It follows from (3) that $\lim _{i} a_{i i}=0$. Hence there is an $i_{0}$ such that for $i>i_{0}, 1 / 2<1 /\left(1+a_{i i}\right)<2$. We put $d_{i}=1$ for $i \leqq i_{0}$ and $d_{i}=1 /\left(1+a_{i i}\right)$ for $i>i_{0}$. Multiplying the $i$ th equation of (1) by $d_{i}$, we obtain

$$
x_{i}+\sum_{k=1}^{\infty} c_{i k} x_{k}=z_{i}, \quad\left(\delta_{i k}+c_{i k}=d_{i}\left(\delta_{i k}+a_{i k}\right) ; z_{i}=d_{i} y_{i}\right)
$$

which has the same solution as (1). Since the $d_{i}$ are bounded and the $c_{i i}=0$ for $i>i_{0}, \sum_{i=1}^{\infty}\left|c_{i i}\right|$ and $\sum_{i=1}^{\infty}\left[\sum_{k=1}^{\infty}\left|c_{i k}\right|^{p /(p-1)}\right]^{p-1}$ converge. It follows that (26) has an absolutely convergent determinant.

II. We solve (26) by Cramer's rule. Let $\Delta^{(j)}$ be the determinant obtained by replacing the $j$ th column of $\Delta=\left|\delta_{i k}+c_{i k}\right|$ by the $z_{i}$ and consider.

$$
\begin{aligned}
\Delta^{(j)}+\sum_{k=1}^{\infty} c_{j k} \Delta^{(k)} & =\sum_{i=1}^{\infty} z_{i} C_{i j}+\sum_{k=1}^{\infty} c_{j k} \sum_{i=1}^{\infty} z_{i} C_{i k} \\
& =\sum_{i=1}^{\infty} z_{i}\left[C_{i j}+\sum_{k=1}^{\infty} c_{j k} C_{i k}\right] \\
& =\sum_{i=1}^{\infty} z_{i} \delta_{i j} \Delta=z_{j} \Delta
\end{aligned}
$$

where $C_{i k}$ is the cofactor of $\delta_{i k} c_{i k}$. This formal procedure is justified if the double series involved converges absolutely. But, from the Hölder inequality, we have 
(28)

$$
\begin{aligned}
& \sum_{k=1}^{\infty}\left|c_{j k}\right| \sum_{i=1}^{\infty}\left|z_{i} C_{i k}\right|=\sum_{k=1}^{\infty}\left|c_{j k}\right|\left\{\left|z_{k} C_{k k}\right|+\sum_{i=1}^{\infty}\left|z_{i} C_{i k}\right|\right\} \\
\leqq & \sum_{k=1}^{\infty}\left|c_{j k}\right|\left\{\left|z_{k} C_{k k}\right|\right. \\
& \left.+\left[\sum_{i=1}^{\infty}\left|z_{i}\right|^{p}\right]^{1 / p}\left[\sum_{i=1}^{\infty}\left|C_{i k}\right| p /(p-1)\right]^{(p-1) / p}\right\} \\
\leqq & C \sum_{k=1}^{\infty}\left|c_{j k} z_{k}\right| \\
& +\left[\sum_{i=1}^{\infty}\left|z_{i}\right|^{p}\right]^{1 / p} \sum_{k=1}^{\infty}\left|c_{j k}\right|\left[\sum_{i=1}^{\infty}\left|C_{i k}\right|^{p /(p-1)}\right]^{(p-1) / p} \\
\leqq & {\left[\sum_{i=1}^{\infty}\left|z_{i}\right|^{p}\right]^{1 / p}\left[\sum_{k=1}^{\infty}\left|c_{j k}\right| p /(p-1)\right]^{(p-1) / p} } \\
& \left\{C+\left(\sum_{k=1}^{\infty}\left[\sum_{i=1}^{\infty}\left|C_{i k}\right| p /(p-1)\right]^{p-1}\right)^{1 / p}\right\},
\end{aligned}
$$

where $C$ is the least upper bound of the $\left|C_{k k}\right|$. The lemma by (5) guarantees the convergence of the double series on the right and hence our series converges absolutely. We have then the solution $x_{k}=\Delta^{(k)} / \Delta$. That the solution is of the required type results as follows, since $\sum_{k=1}^{\infty}\left|x_{k}\right|^{p}=\sum_{k=1}^{\infty}\left|\Delta^{(k)} / \Delta\right|^{p}$ :

$$
\begin{aligned}
\left|\Delta^{(k)}\right| p \leqq & {\left[\sum_{i=1}^{\infty}\left|z_{i} C_{i k}\right|\right]^{p} } \\
= & {\left[\left|z_{k} C_{k k}\right|+\sum_{i=1}^{\infty}\left|z_{i} C_{i k}\right|\right]^{p},(i \neq k \text { on the right }), } \\
& {\left[\sum_{k=1}^{\infty}\left|\Delta^{(k)}\right| p\right]^{1 / p} } \\
\leqq & {\left[\sum_{k=1}^{\infty}\left|z_{k} C_{k k}\right|^{p}\right]^{1 / p}+\left[\sum_{k=1}^{\infty}\left\{\sum_{i=1}^{\infty}\left|z_{i} C_{i k}\right|\right\}^{p}\right]^{1 / p} } \\
\leqq & {\left[\sum_{k=1}^{\infty}\left|z_{k}\right|^{p}\right]^{1 / p}\left(C+\left\{\sum_{k=1}^{\infty}\left[\sum_{i=1}^{\infty}\left|C_{i k}\right|^{p /(p-1)}\right]^{p-1}\right\}^{1 / p}\right) }
\end{aligned}
$$


( $i \neq k$ on the right). The lemma again yields the convergence of the double series on the right.

III. In order to obtain the inverse of (1), we consider the inverse of (26)

$$
z_{k}+\sum_{i=1}^{\infty} \gamma_{i k} z_{i}=x_{k}, \quad\left(\delta_{i k}+\gamma_{i k}=C_{i k} / \Delta\right)
$$

The inverse of (1) then is

$$
\frac{y_{k}}{d_{k}}+\sum_{i=1}^{\infty} \frac{\gamma_{i k}}{d_{i}} y_{i}=x_{k} .
$$

Here $\lim _{i \gamma^{\prime} i i}=0$ and $\sum_{k}^{\infty}{ }_{1}\left[\sum_{i=1}^{\infty}\left|\gamma_{i k}\right|^{p /(p-1)}\right]^{p-1}(i \neq k)$ converges. A set of constants $\delta_{k}\left(1 / 2<\delta_{k}<2\right)$ may be determined as in I so that, in the system

$$
y_{k}+\sum_{i=1}^{\infty} \alpha_{i k} y_{i}=u_{k}
$$

we have

$$
\begin{aligned}
\delta_{i k}+\alpha_{i k} & =\left(\delta_{i k}+\gamma_{i k}\right) \frac{d_{k} \delta_{k}}{d_{i}} ; u_{k}=d_{k} \delta_{k} x_{k}, \\
\alpha_{k k} & =0\left(k>k_{0}\right) ;\left|\alpha_{i k}\right| \leqq \frac{8}{|\Delta|}\left|C_{i k}\right|, \quad(i \neq k) .
\end{aligned}
$$

From (35) it follows that $\sum_{i=1}^{\infty}\left|\alpha_{i i}\right|$ and $\sum_{k=1}^{\infty}\left[\sum_{i=1}^{\infty}\left|\alpha_{i k}\right|^{p /(p-1)}\right]^{p-1}$ converge. Hence the system (33), equivalent to the inverse of (1), has an absolutely convergent determinant.

IV. If (1) is transposed, it becomes

$$
x_{k}+\sum_{i=1}^{\infty} a_{i k} x_{i}=y_{k} .
$$

From (3) it follows that $1 /(p-1) \geqq 1$ and we have, applying the Minkowski inequality if the equality here does not hold,

(37) $\left\{\sum_{k=1}^{\infty}\left[\sum_{i=1}^{\infty}\left|a_{i k}\right|^{p}\right]^{1 /(p-1)}\right\}^{p-1}$

$$
\leqq \sum_{i=1}^{\infty}\left[\sum_{k=1}^{\infty}\left|a_{i k}\right|^{p /(p-1)}\right]^{p-1} .
$$


Therefore $\sum_{i=1}^{\infty}\left|a_{i k}\right|^{p}$ converges for every $k$ and (36) is a transformation on the space $\left(x_{i}\right)$, where $\sum_{i=1}^{\infty}\left|x_{i}\right| p /(p-1)$ converges and $p /(p-1) \geqq 2$. For any such $\left(x_{i}\right)$, we have

$$
\begin{aligned}
{\left[\sum_{k}\left|y_{k}\right|^{p /(p-1)}\right]^{(p-1) / p} \leqq\left[\sum_{i=1}^{\infty}\left|x_{i}\right|^{p /(p-1)}\right]^{(p-1) / p} } \\
\cdot\left[1+\left\{\sum_{k=1}^{\infty}\left[\sum_{i=1}^{\infty}\left|a_{i k}\right|^{p}\right]^{1 /(p-1)}\right\}^{(p-1) / p}\right]
\end{aligned}
$$

and hence the $\left(y_{k}\right)$ is in the same space. On the other hand, given $\left(y_{k}\right)$ in this space, we find an equivalent system (26') as in I having an absolutely convergent determinant and obtain the solution from (26') by Cramer's rule as in II, (6) of the lemma being used. The discussion of the inverse of the transposed system follows that of III.

Princeton University

\section{EXAMPLES OF SURFACE TRANSFORMATIONS}

\section{BY P. A. SMITH}

The purpose of this note is to illustrate by examples certain points in the structure analysis of surface transformations, and at the same time to point out certain unsolved problems which appear to be fundamental in this connection.

Every (1-1) continuous transformation of a surface $S$ into itself admits* a closed invariant set of central points which possesses certain properties of regional recurrence and which is determined essentially as follows: the points of $S$ move under indefinite iteration of $T$ and its inverse $T_{-1}$, toward a certain closed invariant set $M^{1}$ which in general is a proper subset of $S$; $M^{1}$ contains a proper subset $M^{2}$ related to $M^{1}$ as $M^{1}$ is to $S$. Continuing thus, we finally arrive at a first set $M^{r}$ such that

* The definitions and theorems used implicitly in this note are to be found in detail in Birkhoff and Smith, Structure analysis of surface transformations, Journal de Mathématiques, vol. 7 (1928). With regard to regular regions, see also P. A. Smith, Regular components of surface transformations, to appear shortly in the American Journal of Mathematics. 